\title{
Papers
}

\section{Use of magnetic resonance angiography to select candidates with recently symptomatic carotid stenosis for surgery: systematic review}

\author{
Marie E Westwood, Steven Kelly, Elizabeth Berry, John M Bamford , Michael J Gough, C Mark Airey, \\ James F M Meaney, Linda M Davies, Jane Cullingworth, Michael A Smith
}

\begin{abstract}
Objective To determine if sufficient evidence exists to support the use of magnetic resonance angiography as a means of selecting patients with recently symptomatic high grade carotid stenosis for surgery. Design Systematic review of published research on the diagnostic performance of magnetic resonance angiography, 1990-9.

Main outcome measures Performance characteristics of diagnostic test.

Results 126 potentially relevant articles were identified, but many articles failed to examine the performance of magnetic resonance angiography as a diagnostic test at the surgical decision thresholds used in major clinical trials on endarterectomy. 26 articles were included in a meta-analysis that showed a maximal joint sensitivity and specificity of 99\% (95\% confidence interval $98 \%$ to $100 \%$ ) for identifying $70-99 \%$ stenosis and $90 \%(81 \%$ to $99 \%)$ for identifying 50-99\% stenosis. Only four articles evaluated contrast enhanced magnetic resonance angiography.

Conclusions Magnetic resonance angiography is accurate for selecting patients for carotid endarterectomy at the surgical decision thresholds established in the major endarterectomy trials, but the evidence is not very robust because of the heterogeneity of the studies included. Research is needed to determine the diagnostic performance of the most recent developments in magnetic resonance angiography, including contrast enhanced techniques, as well as to assess the impact of magnetic resonance angiography on surgical decision making and outcomes.
\end{abstract}

\section{Introduction}

Patients with arteriosclerosis, particularly those with recent carotid territory stroke or transient ischaemic attack who might benefit from carotid endarterectomy, may be investigated with conventional angiography, ultrasonography, or magnetic resonance angiography. ${ }^{12}$ Magnetic resonance angiography was introduced in the 1980s. The technology continues to evolve rapidly, and three main approaches are currently used: two dimensional time of flight methods, three dimensional time of flight methods, and three dimensional contrast enhanced techniques. Time of flight (or "inflow") methods are non-invasive and emphasise flowing blood because a different signal is given by material that has moved into the imaging volume. Contrast enhanced methods use gadolinium based contrast materials that are better tolerated than the iodinated media used for conventional angiography. Contrast enhancement overcomes some of the drawbacks of time of flight techniques, especially signal loss near stenoses. A greater volume of the body can be imaged in a shorter time, allowing evaluation of the vasculature from the aortic arch to the circle of Willis.

The use of magnetic resonance angiography and ultrasonography has expanded rapidly in recent years, and the use of conventional angiography has declined. Although conventional catheter angiography remains the definitive imaging technique, it is an invasive procedure for which the patient must be admitted to hospital, it involves the use of ionising radiation, and when used in the carotid circulation it is accompanied by serious complications, including a $0.5-2 \%$ risk of stroke. ${ }^{3}$ Magnetic resonance angiography may be more acceptable to patients and may be of particular use in patients not suitable for conventional angiographyfor example, patients with an allergy to iodinated contrast medium, frail and elderly patients, and patients with severe peripheral vascular disease. These potential benefits may be offset by poor performance as a diagnostic test.

This systematic review examines the evidence on the performance of magnetic resonance angiography in evaluating patients with recently symptomatic internal carotid artery stenosis. The North American symptomatic carotid endarterectomy trial (NASCET) and the European carotid surgery trial (ECST) found a clear benefit of surgery in patients with recently symptomatic stenoses of $70-99 \%$ as measured by conventional angiography with the NASCET criteria. ${ }^{4}$ We therefore evaluated the evidence on the diagnostic performance of magnetic resonance angiography in comparison with conventional angiography at this threshold. NASCET and ECST found a smaller benefit of surgery in patients with symptomatic 50-99\% steno-
Academic Unit of
Medical Physics and
Centre of Medical
Imaging Research,
University of Leeds,
Leeds General
Infirmary, Leeds

BMJ 2002;324:1-5 

Economics, University of York, York YO1 5DD Linda M Davies senior research fellow in health economics

Correspondence to: E Berry e.berry@ leeds.ac.uk
Centre for Health

sis; ECST also showed a clear downward trend in the benefit of surgery for stenoses less than $70 \% .{ }^{5}$ The benefit in this group also depended on the age and sex of the patients. However, a recent Cochrane review concluded that surgery was beneficial for patients with $50-69 \%$ stenosis, ${ }^{6}$ so we also evaluated the evidence on the diagnostic performance of magnetic resonance angiography when these more moderate stenoses were included (that is, for patients with 50-99\% stenosis).

For each diagnostic threshold, we aimed to answer the question "What are the sensitivity and specificity of magnetic resonance angiography, in comparison with the gold standard of conventional angiography, in distinguishing severely stenosed arteries suitable for surgery, from either occluded or minimally stenosed arteries?"

\section{Methods}

We sought evaluations of the performance and effectiveness of magnetic resonance angiography in carotid artery stenosis published between January 1990 and December 1999. This review was part of a larger one, in which we also sought articles discussing the use of magnetic resonance angiography in peripheral vessels. We searched the electronic databases Medline, Embase, HealthSTAR, Science Citation Index, Index to Scientific and Technical Proceedings, Cochrane Library, Inside (British Library), and Online Computer Library Centre by using the keywords magnetic resonance angiography and MRA (or accepted synonyms and abbreviations). ${ }^{7}$ We also conducted a hand search of 10 key journals in the fields of imaging and vascular disease. We examined the reference lists of all articles retrieved from the above sources.

We compiled a list of unique articles by eliminating duplicates identified from more than one database. We applied preliminary exclusion criteria within the bibliographic databases, and one reviewer then applied these and a second list of exclusion criteria (table 1). We retrieved the remaining articles, and two reviewers applied final inclusion criteria (table 2) independently, in a hierarchical manner from A to $\mathrm{H}$. Inclusion criteria $\mathrm{A}-\mathrm{C}$ and $\mathrm{F}$ identified articles that described robust primary research comparing the diagnostic performance of magnetic resonance angiography against conventional angiography (A) in terms of sensitivity and specificity (B) and at the specific thresholds of stenosis

Table 1 Number of articles excluded after application of the exclusion criteria; 206 out of 7183 articles remained after these processes, of which 126 were on carotid artery stenosis

\begin{tabular}{lcc} 
Reason for exclusion & $\begin{array}{c}\text { Excluded within } \\
\text { bibliographic databases }\end{array}$ & $\begin{array}{c}\text { Excluded by reviewer (after } \\
\text { exclusions within databases) }\end{array}$ \\
\hline Review & 1230 & 74 \\
\hline Editorial & 123 & 0 \\
\hline Letter & 108 & 6 \\
\hline Case report & 1552 & 0 \\
\hline Conference abstract & 473 & 14 \\
\hline Non-human study & 400 & 7 \\
\hline $\begin{array}{l}\text { Not on magnetic resonance angiography or in } \\
\text { the applications of interest }\end{array}$ & Not applied & 159 \\
\hline Technical evaluation & Not applied & 14 \\
\hline Paediatric participants & Not applied & 123 \\
\hline$\leq 10$ participants & Not applied & 3091 \\
\hline Total No excluded & 3886 &
\end{tabular}

described in our introduction $(\mathrm{C}, \mathrm{F})$. Criterion $\mathrm{D}$ avoids results from the same study being included twice. The remaining criteria are indicators of the validity of the results. The results from articles that did not satisfy criterion E may have had verification bias; if the study group included asymptomatic participants $(G)$ then patient cohort bias may have been present; and disease progression bias could result from a large time period between magnetic resonance angiography and the gold standard $(\mathrm{H}){ }^{8}$ Checklists covering study design, study group characteristics, technical details, and potential biases in executing the study were completed for each article satisfying the inclusion criteria A to D.

We included articles satisfying criteria A-D in the quantitative meta-analysis. Primary data (true positive, true negative, false positive, and false negative values) were extracted independently by two reviewers, and agreed by consensus, for the following clinical decision thresholds measured by using the NASCET criteria or similar" : 70-99\% stenosed vessel (suitable for carotid endarterectomy) versus $0-69 \%$ stenosed or $100 \%$ occluded vessel (not suitable for carotid endarterectomy); $50-99 \%$ stenosed vessel (suitable for carotid endarterectomy) versus $0-49 \%$ stenosed or $100 \%$ occluded vessel (not suitable for carotid endarterectomy). Results derived with the very different ECST criteria were excluded from the meta-analysis. ${ }^{5}$

We plotted results from the independent studies on sensitivity versus 1 -specificity axes to illustrate the scatter of results. We then combined the sensitivity and specificity results for independent studies into a summary receiver operating characteristic curve by using the method recommended by the Cochrane Screening and Diagnostic Tests Methods Working Group for meta-analysis of diagnostic test results. ${ }^{9-12}$ We used a logistic function to transform the sensitivity and specificity data. To prevent undefined values on logistic transformation, we applied a contingency correction of 0.01 to all true positive, false negative, true negative, and false positive values if any one was zero. We fitted a straight line to the transformed results by using an unweighted least squares fit, performed an inverse transformation on the fitted line, and plotted the result as a summary receiver operating characteristic curve.

The summary receiver operating characteristic curve is an excellent graphical summary, but for comparison purposes we calculated a further statistic- $Q^{*}$ and its $95 \%$ confidence interval. ${ }^{9} \mathrm{Q}^{*}$ is the point on the summary receiver operating characteristic curve where sensitivity and specificity have the same value; it represents the maximal joint sensitivity and specificity. $Q^{*}$ is a good summary value in this application as there is no particular disadvantage to sensitivity and specificity being equal: patients with false positive results needlessly undergo the risks of surgery, but patients with false negative results are denied the benefits of surgery. $Q^{*}$ would not be a good choice for describing a screening test, where the aim is to have no false negatives but a few false positives are acceptable.

We then performed a multiple linear regression analysis at the line fitting phase of the summary receiver operating characteristic analysis, to determine if any of five covariates had a significant effect, at the $95 \%$ level, on the fitted summary receiver operating characteristic curve. ${ }^{13}$ The five covariates were tech- 
Table 2 Exclusions on application of final inclusion criteria. Only eight articles remained when all criteria A-H were applied. When only criteria A-D were used, 26 articles satisfied the criteria. ${ }^{16-41}$ References for the excluded articles are available from the authors

\begin{tabular}{llc} 
& Inclusion criterion & No of articles excluded \\
\hline A & Study compared magnetic resonance angiography with digital subtraction angiography or cut film angiography & 32 \\
\hline B & Sufficient data reported for the construction of a $2 \times 2$ contingency table & 44 \\
\hline C & Performance at $50-99 \%$ or $70-99 \%$ or $100 \%$ stenosis reported & 20 \\
\hline D & $\begin{array}{l}\text { Not a duplicate study of same patient group. Where more than one study was found, the one using the largest patient } \\
\text { group was included. }\end{array}$ & 5 \\
\hline E & All patients in study received selective carotid intra-arterial digital subtraction or cut film angiography & 13 \\
\hline F & The method used to determine the degree of stenosis was described & 4 \\
\hline G & No asymptomatic patients were included & 5 \\
\hline H & No time delays of over 1 month occurred between examinations & 1 \\
\hline Total No of articles excluded & 124 \\
\hline
\end{tabular}

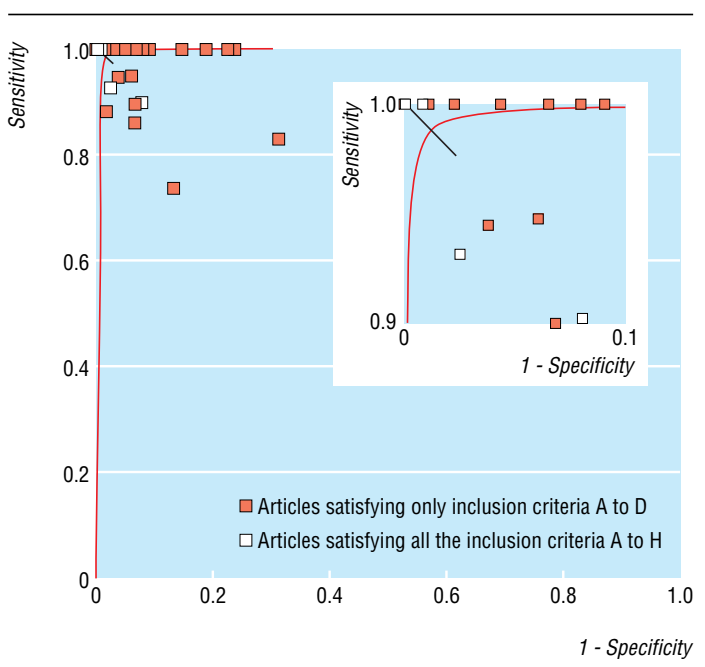

Fig 1 Summary receiver operating characteristic curve for magnetic resonance angiography: $70-99 \%$ stenosis is a positive result, and $0-69 \%$ stenosis and $100 \%$ occlusion are negative results. Straight line shows $95 \%$ confidence interval of $Q^{\star}$. Enlargement of region of sensitivity and specificity $90-100 \%$ shown for clarity

nique of magnetic resonance angiography, inclusion of articles that did not satisfy the inclusion criteria E-H, the risk of test or diagnostic review bias, the risk of verification bias, and the risk of withdrawal bias. ${ }^{14}$ As five variables were tested, the $\mathrm{P}$ value corresponding to $95 \%$ significance was $0.01 .^{15}$ Statistical analyses were performed with SPSS for Windows (release 9.0.0).

\section{Results}

We identified 16185 articles with the initial broad search strategy. After we had removed duplicates, 7183 unique articles remained. The exclusion criteria reduced the number to 206 (table 1), and of 126 candidate articles on carotid artery stenosis 26 satisfied the inclusion criteria A to D. ${ }^{16-41}$ Only eight articles satisfied all the inclusion criteria A to H. ${ }^{172022} 2325353639$ Six of these eight papers included results for the $70-99 \%$ threshold, ${ }^{17} 2223253536$ and two included results for the $50-99 \%$ threshold..$^{20} 39$

For the diagnosis of 70-99\% stenosis (fig 1 ), four sets of results obtained by using contrast enhanced techniques were included, ${ }^{26}{ }^{32} 34{ }^{36}$ together with 11 sets of results (from nine articles ${ }^{19} 23252934353741$ ) obtained by using three dimensional time of flight and 10 sets of results ${ }^{16-38}$ obtained by using two dimensional time of flight techniques. Q* was 99\% (95\% confidence interval
$98 \%$ to $100 \%)$. None of the variables tested in the multiple linear regression, including magnetic resonance angiography technique, was significant at the 95\% level.

For the diagnosis of $50-99 \%$ stenosis (fig 2), no results obtained by using contrast enhanced techniques were included. Results from four studies using three dimensional time of flight techniques were included, ${ }^{21} 293940$ together with six sets of results (from five $\operatorname{articles}^{202428}{ }^{31}{ }^{33}$ ) obtained by using two dimensional time of flight. $Q^{*}$ was $90 \%$ (81\% to $\left.99 \%\right)$. None of the variables tested in the multiple linear regression was significant at the $95 \%$ level.

The patient populations of studies included in the quantitative meta-analysis were heterogeneous. Patient numbers ranged from 11 to $101($ mean $=40)$. In all articles where sex distribution was reported most patients were men; in these articles the proportion of men ranged from $55 \%$ to $100 \%$ (mean $=69 \%)$. Six articles did not report sex distribution. ${ }^{17} 2226293035$ The lower limit for age of patients ranged from 18 to 63 years (mean $=43$ years), and the upper limit ranged from 73 to 87 years (mean $=80$ years). Six articles did not report age range..$^{27} 29343538$ Eight articles stated that asymptomatic patients were included, ${ }^{16} 1827-29313240$ and 18 articles gave no information about symptoms. ${ }^{16} 17$ 19-26 28-30 33-37

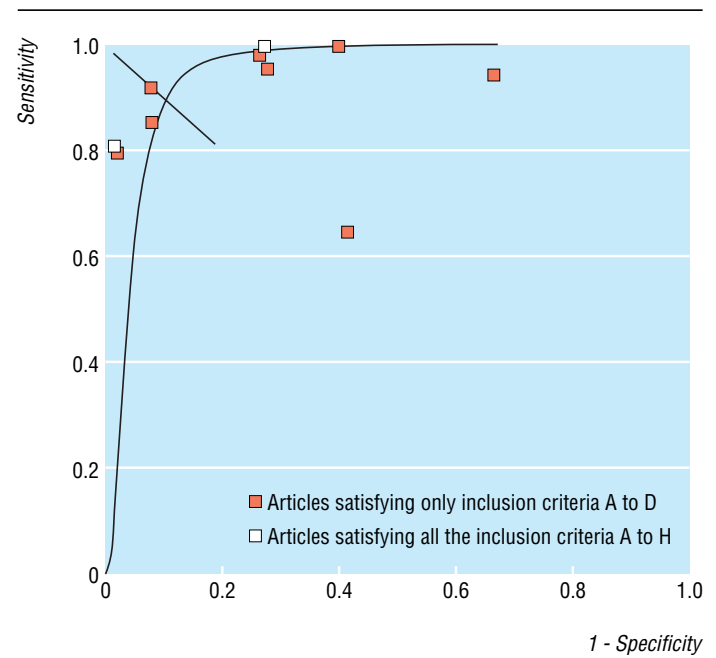

Fig 2 Summary receiver operating characteristic curve for magnetic resonance angiography: $50-99 \%$ stenosis is a positive result, and $0-49 \%$ stenosis and $100 \%$ occlusion are negative results. Straight line shows $95 \%$ confidence interval of $Q^{*}$ 


\section{Discussion}

\section{Available evidence}

Although many articles have been written about magnetic resonance angiography and carotid disease, little rigorous research has been conducted on the performance of magnetic resonance angiography in evaluating carotid artery stenosis. Small numbers of participants and inadequate details of study design mean that many studies included in this review have a potential for bias, but none of the factors tested in the multiple linear regression analysis had a significant effect on the results. Further sources of heterogeneity in patient populations (age, sex distribution, presenting symptoms) may influence patients' suitability for carotid endarterectomy, ${ }^{5}{ }^{42}$ and differences existed between the study groups included in the review, even among those that satisfied all the inclusion criteria.

Potential for bias in this review arose from the increasingly common practice of screening candidates for magnetic resonance angiography by using ultrasonography and proceeding only if this shows a stenosis greater than a predetermined value. Some of the results used in this review were probably obtained in groups of patients preselected in this way, but we were not able to determine this from the articles.

Ultimately, to show effectiveness, we need evidence of the impact of magnetic resonance angiography on clinical decision making and on outcomes in patients. ${ }^{43}$ Comparative studies, with patients randomised to magnetic resonance angiography or conventional angiography, could be used to gather evidence of the impact on decision making. In this review, however, we found no studies that compared magnetic resonance angiography with conventional angiography for surgical decision making or outcomes. Although high quality research is needed, especially full cost effectiveness studies and evaluation of new magnetic resonance angiography techniques, further large trials involving conventional angiography are unlikely to be undertaken. Modelling, using existing evidence, may be the way forward.

\section{Degree of stenosis}

To be able to determine whether a patient is a suitable candidate for carotid endarterectomy, a diagnostic test must distinguish severely $(>70 \%)$ stenosed or moderately $(>50 \%)$ stenosed arteries (which are suitable for carotid endarterectomy) from both minimally stenosed $(0-69 \%$ or $0-49 \%)$ and occluded arteries $(100 \%)$, which are not suitable for carotid endarterectomy. ${ }^{4}$ Candidate articles often failed to assess magnetic resonance angiography in these terms. We excluded 20 articles from the review because they did not classify operable carotid artery stenosis as $70-99 \%$ or $50-99 \%$. Of these, seven articles also failed to report the diagnostic performance of magnetic resonance angiography in separating severely stenosed from occluded arteries. This can be an important distinction in selection for surgery, especially if ultrasonography has indicated a small amount of flow.

Some authors have recommended that the magnitude of the likely benefit of carotid endarterectomy in cases of moderate to severe stenosis should be assessed by using more restricted bands of stenosis. ${ }^{45}$ Future trials with restricted bands might show which stenosis thresholds, as determined by magnetic resonance
What is already known on this topic

Carotid endarterectomy for recently symptomatic carotid stenosis is beneficial in patients with $70-99 \%$ stenosis as measured by conventional angiography

It is not known whether the less invasive imaging technique of magnetic resonance angiography can accurately identify patients who will benefit from surgery

\section{What this study adds}

Magnetic resonance angiography is highly sensitive and specific in diagnosing $70-99 \%$ carotid stenosis

However, the studies on which this conclusion is based are of low quality and high heterogeneity

angiography, are associated with surgical benefit, in addition to determining the reliability of magnetic resonance angiography in detecting patients suitable for surgery. This research might be an ideal testing ground for tracker studies that evaluate fast changing technologies. ${ }^{46}$

\section{Conclusion}

Our review does not support the use of magnetic resonance angiography to select surgical candidates with $50-99 \%$ stenosis. The $95 \%$ confidence interval for $Q^{*}$ extended from $81 \%$ to $99 \%$, and only two of the articles whose results were included in the meta-analysis satisfied the inclusion criteria related to validity. It would be advisable for users of magnetic resonance angiography to ensure that rigorous training and audit are in place, including feedback from surgeons and continuing quality control comparisons with ultrasonography.

Our results indicate that magnetic resonance angiography is very effective for detecting $70-99 \%$ stenosis as defined by conventional angiography. Q* was $99 \%(98 \%$ to $100 \%)$. Although there is a promising trend towards better performance from contrast enhanced methods, further research is essential as only four articles were included in this review and no significant difference was found between the results obtained by using the three main techniques.

We thank all members of the review team for their contributions to the conduct of the systematic review, to hand searching, and to translation.

Contributors: MEW wrote the first draft of the paper and, with SK, performed the literature search and data extraction and participated in designing the review and writing the paper. $\mathrm{EB}$ coordinated the review and the writing of the paper and performed the data analysis. She will be guarantor. JMB, MJG, CMA, JFMM, LMD, and JC participated in the design and execution of the review and in writing the paper. MAS participated in writing the paper.

Funding: Financial support from the secretary of state for health under the NHS Health Technology Assessment Programme (97/13/04). The views and opinions expressed do not necessarily reflect those of the secretary of state for health. Leeds Teaching Hospitals NHS Trust received funding from the NHS Executive; the views expressed in this publication are those of the authors and not necessarily those of the NHS Executive.

Competing interests: JFMM has been reimbursed for presenting material to meetings organised by the pharmaceutical industry (Schering); JFMM has received funds for research on magnetic resonance angiography from both Philips Medical 
Systems and Schering UK; JFMM has received funding from Philips Medical Systems for a part time research assistant; MAS has collaborative links with Philips Medical Systems, who have provided research support for magnetic resonance angiography.

1 Edelman RR. MR angiography: present and future. Am J Roentgenol 1993;161:1-11.

2 Erdoes LS, Marek JM, Mills JL, Berman SS, Whitehill T, Hunter GC, et al. The relative contributions of carotid duplex scanning, magnetic resonance angiography, and cerebral arteriography to clinical decisionmaking: a prospective study in patients with carotid occlusive disease. $J$ Vasc Surg 1996;23:950-6.

3 Davies KN, Humphrey PR. Complications of cerebral angiography in patients with symptomatic carotid territory ischaemia screened by carotid ultrasound.J Neurol Neurosurg Psychiatry 1993;56:967-72.

4 Barnett HJ, Taylor DW, Eliasziw M, Fox AJ, Ferguson GG, Haynes RB, et al. Benefit of carotid endarterectomy in patients with symptomatic moderate or severe stenosis. N Engl J Med 1998;339:1415-25

5 Randomised trial of endarterectomy for recently symptomatic carotid stenosis: final results of the MRC European carotid surgery trial (ECST). Lancet 1998;351:1379-87.

6 Cina CS, Clase CM, Haynes RB. Carotid endarterectomy for symptomatic carotid stenosis. Cochrane Database Syst Reu 2000;(3).CD001081.

7 Berry E, Kelly S, Hutton J, Harris KM, Smith MA. Identifying studies for systematic reviews - an example from medical imaging. Int J Technol Asses Healthcare 2000;16:668-72

8 Kelly S, Berry E, Roderick P, Harris KM, Cullingworth J, Gathercole L, et al. The identification of bias in studies of the diagnostic performance of imaging modalities. Br J Radiol 1997:70:1028-35.

9 Moses LE, Shapiro D, Littenberg B. Combining independent studies of diagnostic test into a summary ROC curve: data-analytic approaches and some additional considerations. Stat Med 1993;12:1293-316.

10 Irwig L, Tosteson AN, Gatsonis C, Lau J, Colditz G, Chalmers TC, et al. Guidelines for meta-analyses evaluating diagnostic tests. Ann Intern Med $1994 ; 120: 667-76$

11 Vamvakas EC. Meta-analyses of studies of the diagnostic accuracy of laboratory tests-a review of the concepts and methods. Arch Pathol Lab Med 1998;122:675-86.

12 Cochrane Methods Working Group on Systematic Review of Screening and Diagnostic Tests. Recommended methods [updated 6 June 1996] http://som.flinders.edu.au/fusa/cochrane/cochrane/sadtdoc1.htm (accessed 9 Nov 2001).

13 Altman DG. Practical statistics for medical research. London: Chapman and Hall, 1991.

14 Reid MC, Lachs MS, Feinstein AR. Use of methodological standards in diagnostic test research. Getting better but still not good. JAMA 1995:274:645-51.

15 Gore SM. Statistics in practice. London: British Medical Association, 1982.

16 Bianchi MC. Magnetic resonance angiography vs digital angiography in surgical selection of carotid stenosis. Nuova Rivista Di Neurologia 1995;5:229-34.

17 Dadachanji MC, Shroff MM, Modi D, Jankharia BG. Comparison of MR angiography with contrast angiography for the diagnosis of carotid artery stenosis. J Assoc Physicians India 1995;43:92-5.

18 Drevet D, Russier S, Age B, Lepine PM, Zabot JM, Joffre P. Study of atheromatous stenoses of carotid bifurcations by Doppler ultrasound, spiral angio-MRI, magnetic resonance angiography and comparison with arteriography.J Radiol 1997;78:1271-7.

19 Fellner C, Strotzer M, Fraunhofer S, Held P, Spies V, Seitz J, et al. MR angiography of the supra-aortic arteries using a dedicated head and neck coil: image quality and assessment of stenoses. Neuroradiology coil: image qual

20 Huston J, Lewis BD, Wiebers DO, Meyer FB, Riederer SJ, Weaver AL Carotid artery: prospective blinded comparison of two-dimensional time-of-flight MR angiography with conventional angiography and duplex US. Radiology 1993;186:339-44.

21 Kido DK, Panzer RJ, Szumowski J, Hollander J, Ketonen LM, Monajati A, et al. Clinical evaluation of stenosis of the carotid bifurcation with magnetic resonance angiographic techniques. Arch Neurol 1991:48:484-9.

22 Laster REJ, Acker JD, Halford HH, Nauert TC. Assessment of MR angiography versus arteriography for evaluation of cervical carotid bifurcation disease. Am J Neuroradiol 1993;14:681-8.

23 Link J, Brinkmann G, Steffens JC, Graessner J, Muller-Hulsbeck S, Heller M. MR angiography of the carotid arteries using $3 \mathrm{D}$ TOF technique with sagittal double-volume acquisition using a new head-neck coil. Rofo Fort schr Geb Rontgenstr Neuen Bildgeb Verfahr 1996;165:544-50

24 Litt AW, Eidelman EM, Pinto RS, Riles TS, McLachlan SJ, Schwartzenberg $\mathrm{S}$, et al. Diagnosis of carotid artery stenosis: comparison of 2DFT time-offlight MR angiography with contrast angiography in 50 patients. Am J Neuroradiol 1991;12:149-54.

25 Magarelli N, Scarabino T, Simeone AL, Florio F, Carriero A, Salvolini U, et al. Carotid stenosis: a comparison between MR and spiral CT angiography. Neuroradiology 1998;40:367-73.

26 Martinat P, Leclerc X, Gauvrit JY, Giboreau F, Pruvo JP. Contribution of fast-sequence three-dimensional MRI angiography with Gadolinium injection in the evaluation of supra-aortic vessels. J Radiol 1998;79:673-

27 Mattle HP, Kent KC, Edelman RR, Atkinson DJ, Skillman JJ. Evaluation of the extracranial carotid arteries: correlation of magnetic resonance angiography, duplex ultrasonography, and conventional angiography. $J$ Vasc Surg 1991;13:838-44

28 Nicholas GG, Osborne MA, Jaffe JW, Reed JF. Carotid artery stenosis: preoperative noninvasive evaluation in a community hospital. J Vasc Surg 1995;22:9-16.

29 Ozaki CK, Irwin PB, Flynn TC, Huber TS, Seeger JM. Surgical decision making for carotid endarterectomy and contemporary magnetic resonance angiography. Am J Surg 1999;178:182-4

30 Pavone P, Catalano C, Di Girolamo M, Albertini PG, Marsili L, Passariello R. Angiography with magnetic resonance of the carotid arteries. Evaluation of clinical results obtained with low magnetic field equipment. Radio Med (Torino) 1993;86:579-86.

31 Polak JF, Bajakian RL, O'Leary DH, Anderson MR, Donaldson MC, Jolesz FA. Detection of internal carotid artery stenosis: comparison of MR angiography, color Doppler sonography, and arteriography. Radiology 1992; 182:35-40.

32 Remonda L, Heid O, Schroth G. Carotid artery stenosis, occlusion, and pseudo-occlusion: first-pass, gadolinium-enhanced, three-dimensiona MR angiography-preliminary study. Radiology 1998;209:95-102.

33 Riles TS, Eidelman EM, Litt AW, Pinto RS, Oldford F, Schwartzenberg GW. Comparison of magnetic resonance angiography, conventiona angiography, and duplex scanning. Stroke 1992;23:341-6.

34 Sardanelli F, Zandrino F, Parodi RC, De Caro G. MR angiography of internal carotid arteries: breath-hold Gd-enhanced 3D fast imaging with steady-state precession versus unenhanced $2 \mathrm{D}$ and $3 \mathrm{D}$ time-of-flight techniques. J Comput Assist Tomogr 1999;23:208-15.

35 Scarabino T, Carriero A, Magarelli N, Florio F, Giannatempo GM, Bonomo L, et al. MR angiography in carotid stenosis: a comparison of three techniques. Eur J Radiol 1998;28:117-25.

36 Scarabino T, Carriero A, Giannatempo GM, Marano R, De Matthaeis P, Bonomo L, et al. Contrast-enhanced MR angiography (CE MRA) in the study of the carotid stenosis: comparison with digital subtraction angiography (DSA).J Neuroradiologie 1999;26:87-91.

37 Sitzer M, Furst G, Fischer H, Siebler M, Fehlings T, Kleinschmidt A, et al. Between-method correlation in quantifying internal carotid stenosis. Stroke 1993;24:1513-8.

38 Turnipseed WD, Kennell TW, Turski PA, Acher CW, Hoch JR. Magnetic resonance angiography and duplex imaging: noninvasive tests for selecting symptomatic carotid endarterectomy candidates. Surgery $1993 \cdot 114 \cdot 643-8$

39 Uehara T, Tabuchi M, Ohsumi Y, Yoneda Y, Mori E. Usefulness of 3-dimensional time-of-flight mr-angiography for evaluation of carotidartery bifurcation stenosis. Cerebrovasc Dis 1995;5:199-203.

40 Wilkerson DK, Keller I, Mezrich R, Schroder WB, Sebok D, Gronlund J, et al. The comparative evaluation of three-dimensional magnetic resonance for carotid artery disease. J Vasc Surg 1991;14:803-9.

41 Young GR, Humphrey PR, Shaw MD, Nixon TE, Smith ET. Comparison of magnetic resonance angiography, duplex ultrasound, and digital subtraction angiography in assessment of extracranial internal carotid artery stenosis. J Neurol Neurosurg Psychiatry 1994;57:1466-78.

42 Bamford J. Risk stratification and carotid surgery: new technology but old trials. Brain 2001;124:455-6.

43 Thornbury JR. Eugene W. Caldwell Lecture. Clinical efficacy of diagnostic imaging: love it or leave it. Am J Roentgenol 1994;162:1-8.

44 Mackenzie R, Dixon AK. Measuring the effects of imaging: an evaluative framework. Clin Radiol 1995;50:513-8

45 Rothwell PM, Gutnikov SA, Eliasziw M, Fox AJ, Taylor W, Mayberg MR, et al. Overall results of a pooled analysis of individual patient data from trials of endarterectomy for symptomatic carotid stenosis. Stroke 2001;32:327.

46 Lilford RJ, Braunholtz DA, Greenhalgh R, Edwards SJ. Trials and fast changing technologies: the case for tracker studies. BMJ 2000;320:43-6.

(Accepted 12 October 2001) 